\title{
Myelin-phagocytosing macrophages modulate autoreactive T cell proliferation
}

\author{
Jeroen FJ Bogie, Piet Stinissen, Niels Hellings and Jerome JA Hendriks
}

\begin{abstract}
Introduction: Multiple sclerosis (MS) is a chronic, inflammatory, demyelinating disease of the central nervous system (CNS) in which macrophages play a central role. Initially, macrophages where thought to be merely detrimental in MS, however, recent evidence suggests that their functional phenotype is altered following myelin phagocytosis. Macrophages that have phagocytosed myelin may be less inflammatory and may exert beneficial effects. The presence of myelin-containing macrophages in CNS-draining lymph nodes and perivascular spaces of MS patients suggests that these cells are ideally positioned to exert an immune regulatory role. Therefore we evaluated in this study the effect of myelin-phagocytosing macrophages on lymphocyte reactivity.

Methods: Thioglycolate-elicited rat peritoneal macrophages were loaded with myelin and cocultured with myelinbasic protein (MBP) or ovalbumin (OVA) reactive lymphocytes. Lymphocyte proliferation was determined by CFSElabeling. The role of nitric oxide in regulating lymphocyte proliferation was assessed by addition of an inhibitor of inducible nitric oxide synthase to the coculture. In vivo immune regulation was investigated by treating MBP-and OVA-immunized animals subcutaneously with myelin. Cognate antigen specific lymphocyte proliferation and nitric oxide production were determined $9 d$ post-immunization.
\end{abstract}

Results: In this study we demonstrate that myelin-phagocytosing macrophages inhibit TCR-triggered lymphocyte proliferation in an antigen-independent manner. The observed immune suppression is mediated by an increase in NO production by myelin-phagocytosing macrophages upon contact with lymphocytes. Additionally, myelin delivery to primarily CD $169^{+}$macrophages in popliteal lymph nodes of OVA-immunized animals results in a reduced cognate antigen specific proliferation. In contrast to OVA-immunized animals, lymphocytes from MBPimmunized animals displayed an increased proliferation after stimulation with their cognate antigen, indicating that myelin-phagocytosing macrophages have dual effects depending on the specificity of surrounding lymphocytes.

Conclusions: Collectively our data show that myelin phagocytosis leads to an altered macrophage function that inhibits lymphocyte proliferation. Additionally, results from this study indicate that myelin-phagocytosing macrophages fulfill a dual role in vivo. On one hand they aggravate autoimmunity by activating myelin-reactive lymphocytes and on the other hand they suppress lymphocyte reactivity by producing NO.

\section{Introduction}

Multiple Sclerosis (MS) is characterized as a chronic, inflammatory, neurodegenerative disease of the central nervous system (CNS). It is regarded to be an autoimmune disease as activated autoimmune lymphocytes are pivotal in orchestrating the immunopathological processes involved in myelin sheath damage [1-4].

Pathologically, MS is characterized by CNS infiltration of activated myelin-reactive lymphocytes and

\footnotetext{
* Correspondence: Jerome.hendriks@uhasselt.be

Hasselt University/Transnational University Limburg, School of Life Sciences, Biomedical Research Institute, Diepenbeek, Belgium
}

macrophages, resulting in an inflammatory microenvironment. Microglia and macrophages typically accumulate in the perivascular spaces and the brain parenchyma near terminal ovoids of transected axons [5]. They are thought to be the primary effector cells in MS and its animal model, experimental allergic encephalomyelitis (EAE) [6-8]. Effector mechanisms of activated macrophages and microglia include the internalization of myelin, and the secretion of inflammatory and toxic mediators which negatively influence axonal and myelin integrity [9-22].

\section{Biomed Central}


In contrast to their apparent detrimental role in MS, increasing evidence suggests an additional neuroprotective role for macrophages. Although two seemingly mutually exclusive processes, various studies have reported such a dual role of monocytes and macrophages in both injury and repair [23,24]. In neurodegenerative models, remyelination is for instance often correlated with large numbers of macrophages and microglia in an inflammatory microenvironment [25-27]. Furthermore, as contact with CNS myelin debris inhibits oligodendrocyte progenitor maturation in vitro, and as macrophages have been described to actively phagocytose myelin debris, local clearance of myelin debris in the centre or vicinity of lesions is suggested to be a necessary prerequisite for axonal remyelination following demyelination [28]. This hypothesis is supported by the fact that monocyte depletion and a consequent inability to clear the microenvironment of myelin debris, causes an impairment of oligodendrocyte progenitor differentiation in vivo $[29,30]$. Finally, recent evidence indicates that monocyte-derived macrophages, peritoneal macrophages, microglia and dendritic cells (DCs) obtain anti-inflammatory characteristics following internalization of myelin [12-14,31]. These studies clearly demonstrate that macrophages, besides their apparent role in neurodegeneration, may exert a neuroprotective influence on MS pathogenesis by clearance of myelin debris and by altering their phenotype following myelin internalization.

Perivascular macrophages, infiltrated macrophages and microglia are ideally positioned to influence infiltrating and infiltrated myelin-reactive lymphocytes. Indeed, CNS reactivation of autoreactive lymphocytes by local antigen presenting cells displaying myelin antigens is thought to initiate and maintain the inflammatory cascade observed in the brain of MS patients [3,4,32]. The presence of brain antigen-containing phagocytes in secondary lymph nodes in MS and EAE further emphasizes a possible crucial role of these cells in modulating the immune response during MS and EAE pathogenesis [33-35]. Phenotypical analysis of these macrophages further revealed that in contrast to neuronal antigen containing phagocytes, the majority of myelin-containing APCs express anti-inflammatory mediators. How brain antigens gain excess to CNS draining secondary lymph nodes, either chemotactically in the context of phagocytes or as soluble products, remains to be clarified [12,36,37].

In this study we investigated the capacity of myelinphagocytosing macrophages (mye-macrophages) to influence lymphocyte proliferation. We show that myemacrophages inhibit TCR-triggered lymphocyte proliferation in an antigen-independent manner. This process is mediated by an enhanced nitric oxide (NO) production. Furthermore, we demonstrate that myelin delivery to popliteal lymph nodes of OVA-immunized animals and uptake by primarily $\mathrm{CD} 169^{+}$macrophages reduces cognate antigen specific proliferation following restimulation ex vivo. The elevated production of $\mathrm{NO}$ detected in these lymph node cultures indicates that NO may also mediate the immune suppressive effects in vivo. In contrast, myelin delivery to popliteal lymph nodes did increase lymphocyte reactivity in MBP-immunized animals. Thus, mye-macrophages may play a suppressive role in CNS-draining lymph nodes during MS pathogenesis, depending on the nature of surrounding lymphocytes. Collectively our data provide evidence that myelin phagocytosis leads to an altered macrophage function that modulates lymphocyte responses.

\section{Methods \\ Animals}

Female Lewis rats, 6-8 weeks of age, were purchased from Harlan Netherlands B.V. (Horst, The Netherlands). Animals were housed in the animal facility of the Biomedical Research Institute of Hasselt University. Experiments were conducted in accordance with institutional guidelines and approved by the local Ethical Committee for Animal Experiments of Hasselt University.

\section{Isolation of peritoneal rat macrophages}

Three days prior to macrophage isolation, rats were injected intraperitoneally with $3 \mathrm{ml} \mathrm{3 \%}$ thioglycolate (Sigma-Aldrich, Bornem, Belgium). Resident peritoneal macrophages were obtained by peritoneal lavage using $10 \mathrm{ml}$ of ice-cold PBS (Lonza, Vervier, Belgium) supplemented with $5 \mathrm{mM}$ ethylenediamine tetraacetic acid (EDTA; VWR, Leuven, Belgium). Peritoneal exudate cells (PECs) were cultured for 2 hours in RPMI 1640 medium. After 2 hours incubation at $37^{\circ} \mathrm{C}$ with $5 \% \mathrm{CO}_{2}$, non-adherent cells were washed away. Remaining cells were $>95 \%$ macrophages [38].

\section{Myelin phagocytosis}

Myelin was purified from rat brain tissue by means of density-gradient centrifugation, as described previously [39]. Myelin protein concentration was determined by using the BCA protein assay kit (Thermo Fisher Scientific, Erembodegem, Belgium). LPS content was determined using the Chromogenic Limulus Amebocyte Lysate assay kit (Genscript Incorperation, Aachen, Germany). Isolated myelin contained a neglectable amount of endotoxin $\left(1.8 \times 10^{-3} \mathrm{pg} / \mu \mathrm{g}\right.$ myelin $)$.

Isolated myelin was fluorescently labelled, according to the method of Van der Laan et al. [11]. In short, $10 \mathrm{mg} /$ $\mathrm{ml}$ myelin was incubated with $12.5 \mu \mathrm{g} / \mathrm{ml} \mathrm{1,1"-diotade-}$ cyl-3,3,3',3',-tetramethylindocarbocyanide perchlorate (DiI; Sigma-Aldrich) for $30 \mathrm{~min}$ at $37^{\circ} \mathrm{C}$. Next, a myelin 
phagocytosis assay was performed as previously described [39].

\section{Immunization and in vivo myelin treatment}

Lewis rats were injected subcutaneously with a $0.1 \mathrm{ml}$ suspension containing $250 \mu \mathrm{g} / \mathrm{ml}$ guinea pig myelin basic protein (MBP) or ovalbumin (OVA), $2.5 \mathrm{mg} / \mathrm{ml}$ H37RA heat-killed mycobacterium tuberculosis (Difco, Detroit, USA) and $60 \mu \mathrm{l}$ Complete Freunds adjuvant (Sigma-Alldrich) in both hind paws. Subsequently animals were injected subcutaneously with PBS, $2.6 \times 10^{6}$ latex beads $(0.8 \mu \mathrm{m}$ mean particle size, Sigma-Alldrich), $75 \mu \mathrm{g} /$ animal of isolated myelin or OVA (d-4, 0,4 and 8 pre- and post-immunization). MBP-immunized rats were weighted and scored daily according to the following neurological scale: $0=$ no neurological abnormalities, $0.5=$ partial loss of tail tonus, $1=$ complete loss of tail tonus, $2=$ hind limb paresis, $3=$ hind limb paralysis, $4=$ moribund, $5=$ death.

\section{Generation of antigen-specific lymphocytes}

MBP and OVA-specific lymphocytes were obtained 9 days post-immunization by bilateral isolation of the inguinal and popliteal lymph nodes. Single-cell suspensions of harvested lymph nodes were obtained by grinding with a syringe plunger against a $70 \mu \mathrm{m}$ cell strainer (Bellco Glass Inc., Vineland, USA). To enrich for antigen specific lymphocytes, lymph node cells were restimulated, as described previously [40]. Briefly, lymph node cells were resuspended in stimulation medium: RPMI 1640 medium (Invitrogen, Merelbeke, Belgium) supplemented with $50 \mathrm{U} / \mathrm{ml}$ penicillin (Invitrogen), $50 \mathrm{U} / \mathrm{ml}$ streptomycin (Invitrogen), $20 \mu \mathrm{M} 2$-mercapto-ethanol (Sigma-Alldrich), 1\% sodium pyruvate (Invitrogen), 1\% MEM non-essential amino acids (Invitrogen), 2\% deactivated autologous serum and $33 \mu \mathrm{g} / \mathrm{ml}$ MBP. After 2 days, cells were washed and resuspended in RPMI 1640 medium supplemented with $50 \mathrm{U} / \mathrm{ml}$ penicillin, $50 \mathrm{U} / \mathrm{ml}$ streptomycin, $20 \mu \mathrm{M}$ 2-mercapto-ethanol, 10\% fetal calf serum (FCS, Hyclone, Erembodegem, Belgium) and 6,5\% supernatants of Concanavalin (ConA, Sigma-Alldrich) stimulated spleen cells. Following 2 days, cells were washed and resuspended in RPMI 1640 medium supplemented with $50 \mathrm{U} / \mathrm{ml}$ penicillin, $50 \mathrm{U} / \mathrm{ml}$ streptomycin, $20 \mu \mathrm{M} 2$ 2-mercapto-ethanol and 10\% fetal calf serum for 3 days.

\section{CFSE-labeling of lymphocytes}

A carboxyfluorescein diacetatesuccinimidyl ester (CFSE) stock (10 mM in DMSO, Invitrogen, Merelbeke, Belgium) was diluted in PBS (Biowhittaker ${ }^{\mathrm{TM}}$ ). Antigen-specific lymphocytes were resuspended in PBS supplemented with $0.05 \%$ BSA and $4 \mu \mathrm{M}$ CFSE $(20 \times$ $10^{6}$ cells $/ \mathrm{ml}$ ) for $7 \mathrm{~min}$ at $37^{\circ} \mathrm{C}$ with $5 \% \mathrm{CO}_{2}$. Cells were washed and diluted in $0.5 \mathrm{ml}$ culture medium for 30 min at $37^{\circ} \mathrm{C}$ with $5 \% \mathrm{CO}_{2}$ to stabilize the CFSE-labeling. In parallel, to determine macrophage viability following a coculture with lymphocytes, macrophages were labeled with CFSE to distinguish them from unlabeled lymphocytes.

\section{Coculture of macrophages with lymphocytes}

Prior to coculture with CFSE labeled lymphocytes, isolated macrophages were seeded in flat-bottem 96-well plates $\left(15 \times 10^{3}\right.$ cells/well $)$ in RPMI 1640 medium supplemented with $50 \mathrm{U} / \mathrm{ml}, 50 \mathrm{U} / \mathrm{ml}$ streptomycin and $10 \% \mathrm{FCS}$, and treated with $100 \mu \mathrm{g} / \mathrm{ml}$ of isolated myelin for three hours. Excess myelin was removed by washing twice with RPMI 1640 medium at $37^{\circ} \mathrm{C}$. Subsequently, stimulation medium containing irradiated thymocytes $\left(15 \times 10^{4}, 3000 \mathrm{rad}\right)$, CFSE-labeled MBP- or OVA-specific lymphocytes $\left(15 \times 10^{4}\right)$ and respectively $10 \mu \mathrm{g} / \mathrm{ml}$ MBP or $10 \mu \mathrm{g} / \mathrm{ml}$ OVA were added. Untreated macrophages were used as a control. To evaluate the involvement of respectively $\mathrm{NO}$, arginase, indoleamine 2,3dioxygenase (IDO) the phagocytosis process itself, direct cell-cell contact and IFN $\gamma, 1.5 \mathrm{mM} \mathrm{N}^{\mathrm{G}}$-Monomethyl-Larginine (L-NMMA; VWR), $0.5 \mathrm{mM} \mathrm{N}^{\mathrm{G}}$-Hydroxy-Larginine (NOHA; VWR), $0.2 \mathrm{mM}$ 1-Methyl-L-tryptophan (1-MT; Sigma Alldrich), latex beads (1:100), 100 $\mu \mathrm{g} / \mathrm{ml}$ zymosan A (Sigma-Alldrich), transwell inserts (0.4 $\mu \mathrm{m}$ pore size, Sigma-Aldrich) or $10 \mu \mathrm{g} / \mathrm{ml}$ anti-rat IFN $\gamma$ (Preprotech, London, UK) were tested in the coculture model.

Flow cytometry was used to assess proliferation and cell death of lymphocytes and macrophages after a 4 day coculture. Here, cells were stained with PE-conjugated mouse-anti-rat CD3 (Immunosource, Erembodegem, Belgium) or CD11b (AbD Serotec, Düsseldorf, Germany) and 7 aminoactinomycin D (7AAD, BD Biosciences).

\section{$\left[{ }^{3} \mathrm{H}\right]$ Thymidine incorporation}

Isolated lymph node cells $\left(20 \times 10^{4}\right)$ were cultured with MBP $(10 \mu \mathrm{g} / \mathrm{ml})$, OVA $(10 \mu \mathrm{g} / \mathrm{ml})$ or myelin-oligodendrocyte glycoprotein (MOG, $20 \mu \mathrm{g} / \mathrm{ml}$ ). Additionally, $100 \mu \mathrm{g} / \mathrm{ml}$ of isolated myelin was added in some experiments. Following $48 \mathrm{hr}, 1 \mu \mathrm{Ci}\left[{ }^{3} \mathrm{H}\right]$ thymidine (Amersham, Buckinghamshire, UK) was added to the culture. Next, cells were harvested with an automatic cell harvester (Pharmacia, Uppsala, Sweden) and uptake of radioactivity was measured in a $\beta$-plate liquid scintillation counter (Wallac, Turku, Finland).

\section{Nitrite formation}

Coculture supernatants were collected and release of NO was determined using the griess reagent system (Promega, Leuven, Belgium), following the manufacturer's 
instructions. Absorbance was determined by using a microplate reader at $550 \mathrm{~nm}$ (Biorad Benchmark).

\section{Histology and immunohistochemistry}

Snap-frozen brain and spinal cord material was cut in respectively the coronal and sagittal plane with a Leica CM1900UV cryostat (Leica Microsystems, Wetzlar, Germany) to obtain $10 \mu \mathrm{m}$ sections. The extent of demyelination and infiltration was determined by staining with Luxol Fast Blue (LFB; Gurr BDH, Poole, England). Briefly, aceton-fixed slides were incubated with LFB for $16 \mathrm{hr}$ at $56^{\circ} \mathrm{C}$, destained with $0.05 \%$ lithium carbonate, and counterstained with cresyl violet (VWR). Analysis was carried out using a Nikon eclipse 80i microscope and NIS Elements BR 3.10 software (Nikon, Tokyo, Japan).

DiI-labeled myelin migration to popliteal and inguinal lymph nodes was determined by immunohistochemistry. Popliteal and inguinal lymph nodes were snap-frozen directly following isolation and cut into $10 \mu \mathrm{m}$ sections. Following fixation in respectively aceton for $10 \mathrm{~min}$, sections and cells were blocked using $10 \%$ goat serum (Millipore, Brussels, Belgium) in PBS. Subsequently, sections and cells were stained with mouse-anti-rat CD169 (1/250 in PBS; Abd Serotec), a marker for macrophages in lymph nodes. As a secondary antibody Alexa fluor $488 \mathrm{~F}(\mathrm{ab})_{2}$ fragment of goat-anti mouse was used (1/ 500 in PBS; Invitrogen). Control staining was performed by omitting the primary antibody. Nuclear staining was performed using 4,6'-diamidino-2-phenylindole (DAPI; Invitrogen) for $10 \mathrm{~min}$. Autofluorescence was minimalized by using 0.1\% Sudan Black in 70\% ethanol.

\section{Statistical analysis}

Data were statistically analyzed using GraphPad Prism for windows (version 4.03) and are reported as mean \pm SEM. D'Agostino and Pearson omnibus normality test was used to test normal distribution. An analysis of variances (ANOVA) or two-tailed unpaired student T-test (with Welch's correction if necessary) was used for normally distributed data sets. The Kruskal-Wallis or Mann-Whitney analysis was used for data sets which did not pass normality. ${ }^{*} \mathrm{P}<0,05,{ }^{* *} \mathrm{P}<0,01$ and ${ }^{* * *} \mathrm{P}<$ 0,001 .

\section{Results \\ Myelin-laden macrophages inhibit lymphocyte proliferation}

Initially we assessed the capacity of peritoneal macrophages to internalize myelin. By culturing macrophages with different concentrations of DiI-labeled myelin for divergent periods of time, it was demonstrated that myelin is internalized in a time- and dose-dependent manner (Figure 1a). Next, the most optimal macrophage/ lymphocyte coculture ratio was determined by using untreated macrophages. At high macrophage/lymphocyte ratio's, lymphocytes demonstrated a reduced viability and proliferation (Figure 1b and 1c). The decline of lymphocyte viability was absent at low macrophage/lymphocyte ratio's $(<1 / 10)$. To observe differences in lymphocyte proliferation following coculture with mye-macrophages, subsequent experiments were conducted using a macrophage/lymphocyte ratio of $1 / 10$.

To study whether mye-macrophages affect antigenspecific lymphocyte proliferation in a different manner compared to untreated macrophages, macrophages or mye-macrophages were cocultured for $4 \mathrm{~d}$ with MBP- or OVA-reactive lymphocytes, irradiated thymocytes and purified MBP or OVA. Here it was demonstrated that mye-macrophages inhibit TCR-triggered lymphocyte proliferation more pronounced than untreated macrophages (Figure 1d and 1e). This process was independent of antigen-specificity, since both MBP and OVAreactive lymphocytes showed the same reduction in proliferation. Proliferation differences were not due to an altered viability of lymphocytes or mye-macrophages (data not shown).

Inhibition of lymphocyte proliferation is independent of the phagocytosis process or myelin-antigen presentation

To elucidate the mechanisms behind the increased inhibition of lymphocyte proliferation by mye-macrophages, we assessed whether the phagocytosis process as such is responsible for the observed effects on lymphocyte proliferation. For this purpose, macrophages were loaded for 3 consecutive hours with latex beads or zymosan A prior to coculture with lymphocytes. Macrophage treatment with beads or zymosan significantly affected their capacity to modulate lymphocyte proliferation compared to control and myelin treated macrophages (Figure 2a), indicating that the observed increased inhibition of lymphocyte proliferation relies on myelin-specific effects, instead of being induced by the phagocytosis process itself.

Like DCs, macrophages can act as messengers of innate and adaptive immunity by presenting antigen in context of MHC molecules. Mye-macrophages can therefore be assumed to process endogenous myelin and present it to myelin-reactive lymphocytes, possibly influencing reactivity of these lymphocytes. To determine whether the observed immune suppressive effects are dependent on antigen presentation, untreated macrophages and mye-macrophages were cocultured with MBP-reactive lymphocytes in the absence of MBP and irradiated thymocytes. Mye-macrophages did not affect lymphocyte proliferation (Figure $2 \mathrm{~b}$ ), suggesting that, at a macrophage/lymphocyte ratio of $1 / 10$, mye-macrophages do not influence the proliferation of MBP- 


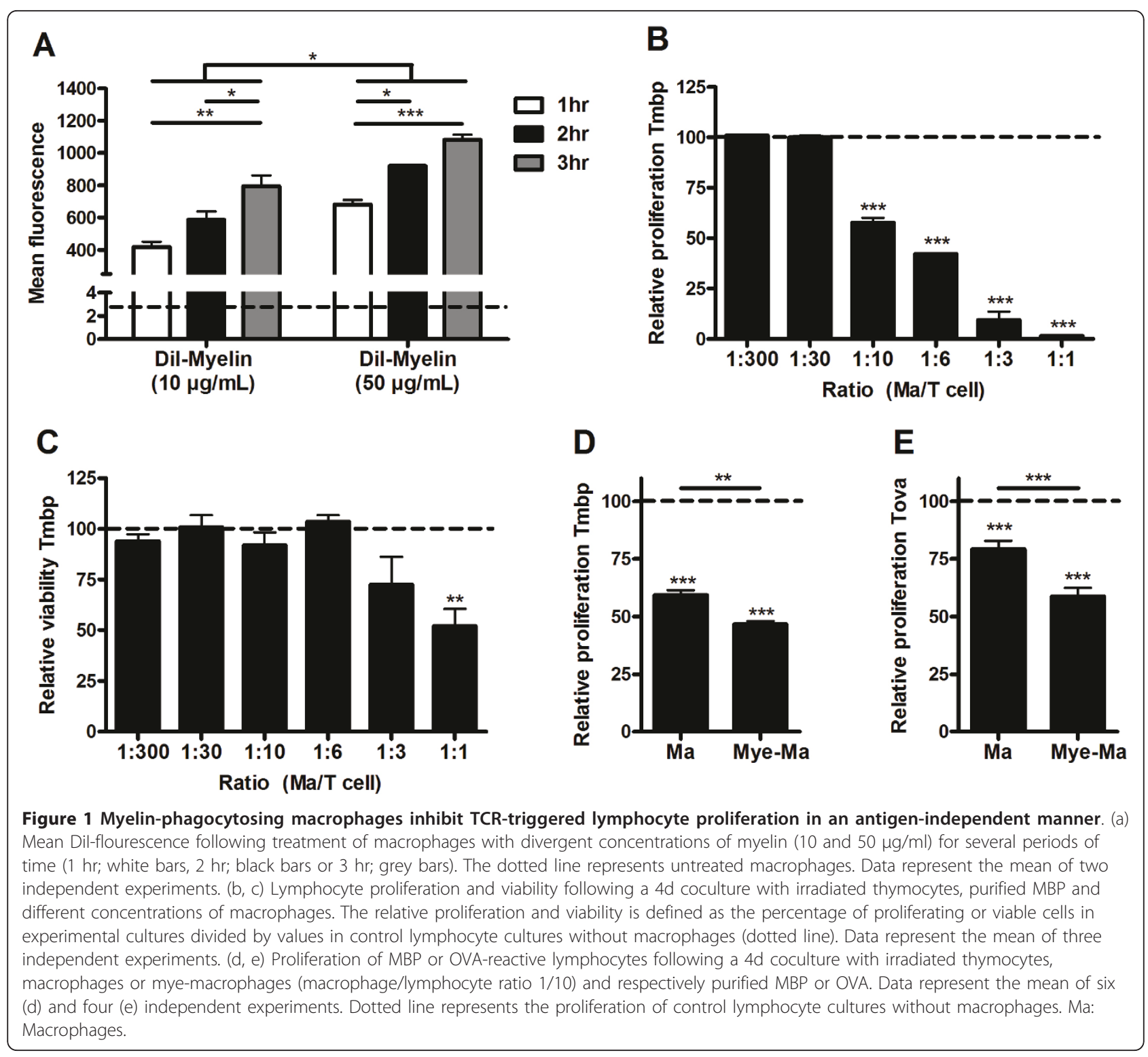

reactive lymphocytes. These results are in line with the observed equal inhibition of proliferation of both MBP and OVA-reactive lymphocyte by mye-macrophages.

Lipid components of myelin, like cholesterol and arachidonic acid-containing phosphatidylcholine, have been reported to directly inhibit proliferation of ConA stimulated lymphocytes [41,42]. Accordingly, we assessed whether isolated myelin has a direct effect on lymphocyte proliferation. By culturing lymphocytes with increasing concentrations of isolated myelin, in the absence of macrophages, it was established that myelin itself, even at high concentrations, had no significant influence on lymphocyte reactivity (data not shown).

Together, these data indicate that direct effects of myelin on lymphocyte proliferation, myelin-antigen presentation and the phagocytosis process itself are not responsible for the observed effects on lymphocyte proliferation.

\section{Macrophages inhibit lymphocyte proliferation by the} production of nitric oxide

Macrophages have been reported to inhibit lymphocyte proliferation in vitro by mechanisms involving inducible nitric oxide synthase (iNOS), arginase I or IDO [43-55]. By administrating either an inhibitor of NOS (LNMMA), arginase (NOR-NOHA) or IDO (1-MT) to the culture, the involvement of these enzymes was evaluated. Here we demonstrated that an increased activity of both IDO and arginase did not account for the increased inhibition of lymphocyte proliferation by mye- 


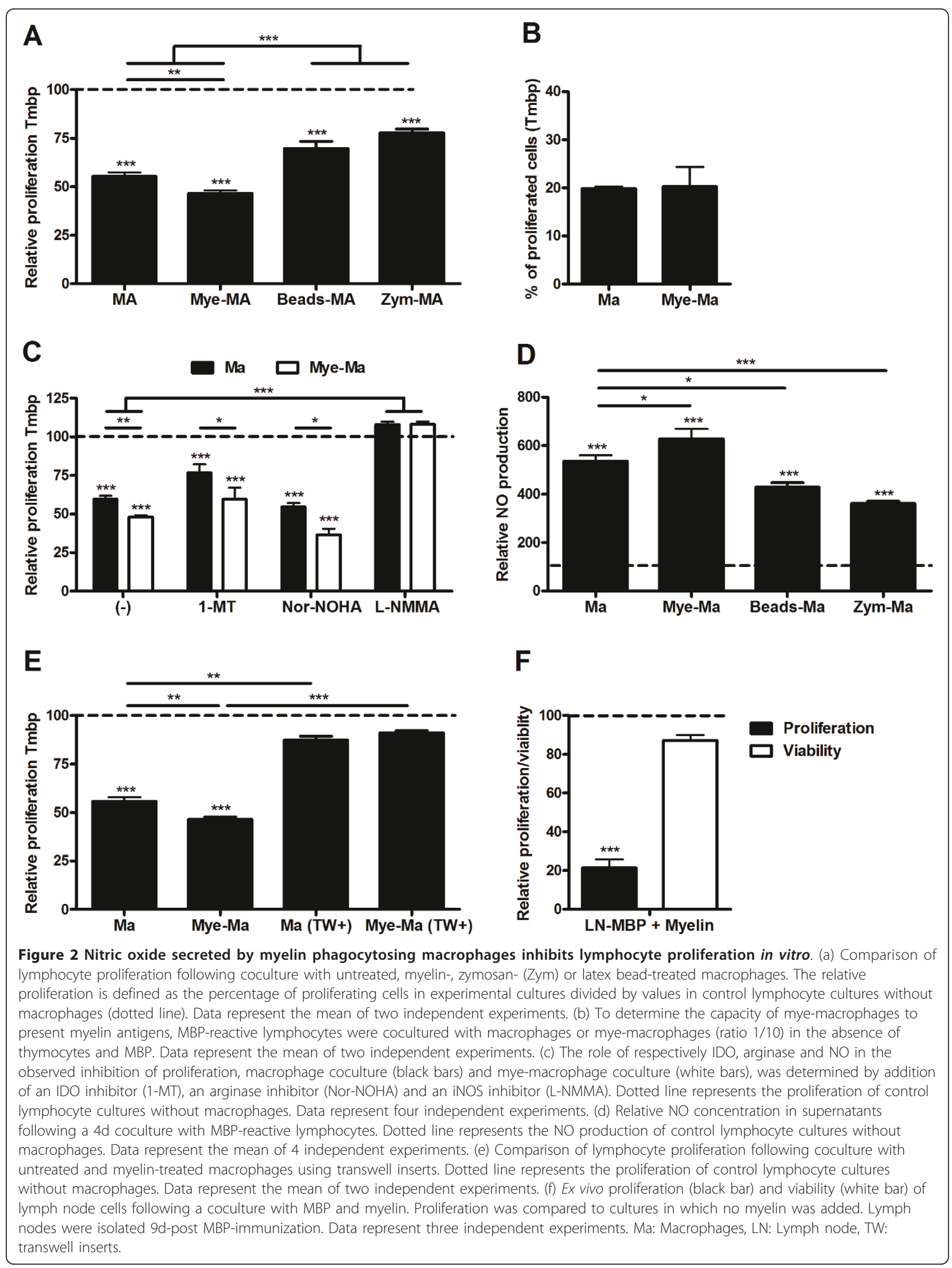


macrophages (Figure 2c). In contrast, administration of L-NMMA completely abrogated the inhibition of lymphocyte proliferation by both macrophages and myemacrophages. Yet again, cocultures of both OVA and MBP-reactive lymphocytes were affected equally (data not shown). The importance of $\mathrm{NO}$ was further delineated by assessment of NO levels in the coculture supernatant of MBP-reactive lymphocytes. In contrast to cocultures of lymphocytes with latex beads or zymosan treated macrophages, a significantly increased concentration of NO was observed in the supernatants derived from cocultures with mye-macrophages, when compared to untreated macrophages (Figure $2 \mathrm{~d}$ ). This increased NO production by macrophages following myelin internalization was absent in monocultures (data not shown), indicating that the observed increased concentration of $\mathrm{NO}$ in the coculture with mye-macrophages was induced by lymphocytes. Lymphocyte-derived IFN $\gamma$ has previously been described to induce NO production in macrophages [56]. However, in our coculture model no increase in IFN $\gamma$ in the supernatants of cocultures with mye-macrophages compared to untreated macrophages was found (data not shown). Correspondingly, IFN $\gamma$ neutralization did neither abrogate the increased inhibition of proliferation by mye-macrophages or decrease the NO production in cocultures (data not shown). Nonetheless, when transwell inserts were used to restrict direct cell-cell contact, lymphocyte proliferation in cocultures of both untreated or myelin-treated approached control values (Figure $2 \mathrm{e}$ ), indicating a role for direct cell-cell contact in the induction of NO.

Noteworthy, when lymph node cells, isolated 9 days post-immunization, were exposed directly to MBP and myelin, an even more pronounced myelin-mediated inhibition of lymphocyte proliferation was observed (Figure $2 \mathrm{f}$ ). The latter indicates that local lymph node phagocytes show a similar immune suppressive response as peritoneal macrophages following myelin ingestion. Differences detected in proliferation were not due to an altered viability of lymphocytes (Figure $2 \mathrm{f}$ ).

\section{Myelin modulates lymph node proliferation in vivo}

Since we established that myelin internalization by macrophages alters their capacity to modulate $\mathrm{T}$ cell proliferation in vitro, we assessed the in vivo suppressive capacity of mye-macrophages. First, to determine whether subcutaneous injected myelin reaches the draining lymph node and is taken up by macrophages, we injected DiI-labeled myelin in the footpad of healthy animals. A notable migration of myelin towards popliteal lymph nodes was observed (Figure 3a). Immunohistochemical analysis further revealed that myelin was contained primarily in $\mathrm{CD} 169^{+}$macrophages located at
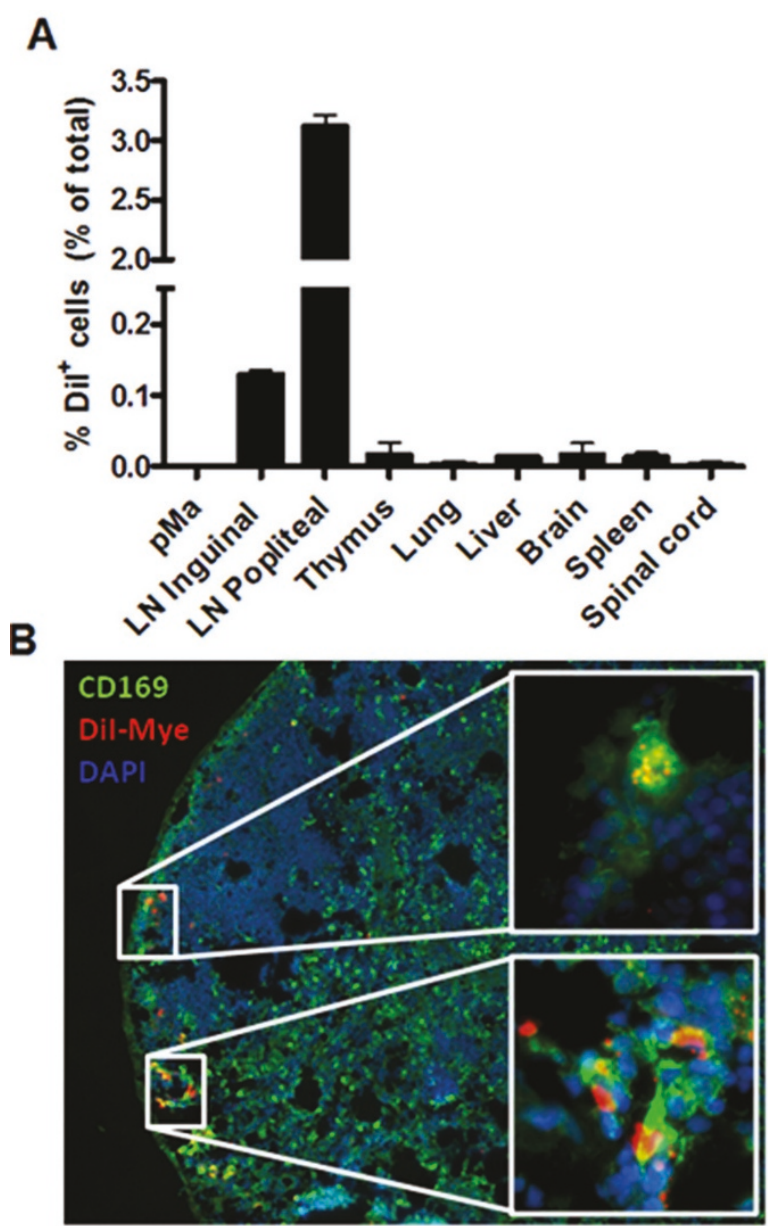

Figure 3 Myelin-mediated inhibition of lymph node proliferation and myelin migration to $\mathrm{CD} 169^{+}$macrophages in popliteal lymph nodes. (a) Dil-labeled myelin was injected subcutaneously in the footpad of healthy animals. Dil-flourescene was determined $4 \mathrm{~d}$ post-injection by flow cytometry. One experiment is shown. (b) Immunohistochemical staining of popliteal lymph nodes $4 \mathrm{~d}$ post-injection of Dil-labeled myelin. Sections were additionally stained with CD169 and DAPI. pMa: Peritoneal macrophages, LN: Lymph node.

the border of the medulla and in the subcapsular sinus (Figure 3b).

Next, OVA-immunized animals were treated subcutaneously in the footpad with myelin (d-4, 0, 4, 8 pre/ post-immunization). Recall stimulation in vitro, $9 \mathrm{~d}$ postimmunization, revealed a reduced cognate antigen specific proliferation in animals treated with myelin (Figure 4a). Interestingly, LPS-stimulated lymph node cultures from myelin-treated animals demonstrated an increased NO production (Figure 4b). These results demonstrate that myelin is capable of suppressing antigen-specific proliferation in vivo and suggest that an increased NO production by these cells is responsible for this effect. 

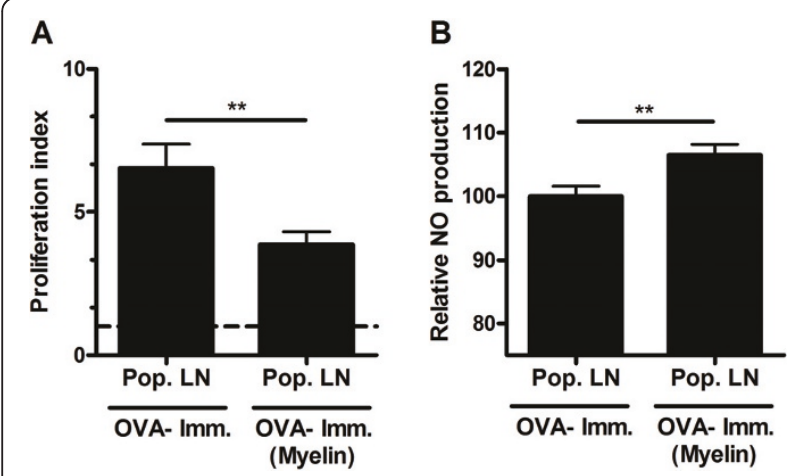

Figure 4 Myelin inhibits lymphocyte proliferation in OVAimmunized animals. (a) OVA-immunized animals ( $N=7$ ) were treated with myelin at day $-4,0,4$ and 8 (or left untreated). Nine days post-immunization, OVA-reactivity of isolated lymph node cultures was assessed. Non-stimulated cultures were used as control. Data represent the mean of seven independent experiments. (b) NO production by LPS-stimulated popliteal lymph node cultures $9 d$ post-immunization. Lymph node cultures were stimulated with LPS for $18 \mathrm{hr}$ after which NO production in the supernatant was determined. Data represent the mean of seven independent experiments. Pop. LN: Popliteal lymph node.

In contrast to OVA-immunized animals, MBP-immunized animals revealed an increased reactivity towards MBP and MOG following myelin treatment (Figure 5a). Moreover, myelin-treated animals demonstrated an earlier onset of paralysis and a more severe neurological score at the peak of disease (Figure $5 \mathrm{~b}$ ). In concordance, a LFB/cresyl violet staining of brain and spinal cord sections demonstrated increased cellular infiltrates and the presence of demyelinated areas in myelin-treated animals (Figure 5c). Noteworthy, when MBP-immunized animals were treated with latex beads no overt effect on disease score and onset was found (data not shown), indicating that the aggravated disease score following myelin treatment is myelin-specific.

To exclude the possibility that in the restricted area of the lymph node the availability of OVA in OVA-immunized animals is altered by the additional presence of myelin proteins, hereby reducing OVA-specific proliferation, MBP-immunized animals were treated subcutaneously with OVA. As expected, an increased reactivity towards OVA was observed in isolated lymph node cells (Figure 6a). However, MBP-specific proliferation was unchanged (Figure 6b), demonstrating that the observed myelin-mediated inhibition of proliferation of lymph nodes cultures from OVA-immunized is independent of a reduced availability of OVA by myelin proteins.

These results indicate that mye-macrophages have a dual influence on proliferation, depending on the nature of surrounding lymphocytes. On one hand they aggravate autoimmunity by activating myelin-reactive lymphocytes and on the other hand they have the capacity to suppress lymphocyte activation by the secretion of NO.

\section{Discussion}

In this study we have established that macrophages that have phagocytosed myelin modulate the proliferation of autoreactive $\mathrm{T}$ cells. The observed inhibition of TCRtriggered lymphocyte proliferation by mye-macrophages was antigen-independent, as both OVA- and MBP-reactive lymphocytes show an identical reduction in proliferation following coculture with mye-macrophages in vitro. Additionally, when in vivo primed lymph node cultures were restimulated directly in vitro in the presence of myelin, an even more pronounced immune suppression was observed. These results indicate that both macrophages and lymph node phagocytes obtain immune suppressive properties following myelin internalization.

Macrophages may inhibit proliferation of lymphocytes in various manners, including IDO-mediated depletion of tryptophan, arginase-mediated lowering of L-arginine and lymphocyte $\mathrm{CD} 3 \zeta$ expression, and NO-mediated reduction of tyrosine residue phosphorylation in the Jak3/STAT5 pathway and inhibition of caspase activity [43-52]. We demonstrate that the non-selective iNOS inhibitor L-NMMA completely reversed the observed inhibition of proliferation by both control and myemacrophages while the other pathways were not involved. In line with this, an increased concentration of $\mathrm{NO}$ was demonstrated in the coculture supernatant of mye-macrophages, explaining the observed inhibition of lymphocyte proliferation by mye-macrophages.

Abrogation of direct cell-cell contact restored lymphocyte proliferation in our cocultures. This finding, together with the observed role of NO in the inhibition of lymphocyte proliferation, suggests that direct contact between both cell types is a necessary prerequisite for stimulating NO-mediated inhibition of lymphocyte proliferation by macrophages. On the other hand, NO might, due to extreme short half-life, not reach lymphocytes when direct contact is restricted. Future studies should therefore determine the mechanism behind the macrophage- and mye-macrophage-mediated inhibition of lymphocyte proliferation in our cocultures. Although lymphocyte-derived IFN $\gamma$ is described to induce NO production by macrophages, we were unable to demonstrate a role for lymphocyte-produced IFN $\gamma$ in the observed inhibition of lymphocyte proliferation [56].

As we demonstrated an increased, NO-mediated inhibition of lymphocyte proliferation by mye-macrophages in vitro, myelin-rich phagocytes in secondary lymph nodes might fulfill an identical suppressive role in vivo. $\mathrm{CD} 169^{+}$macrophages in lymph nodes are described to be primarily involved in uptake and relay of viral 
A

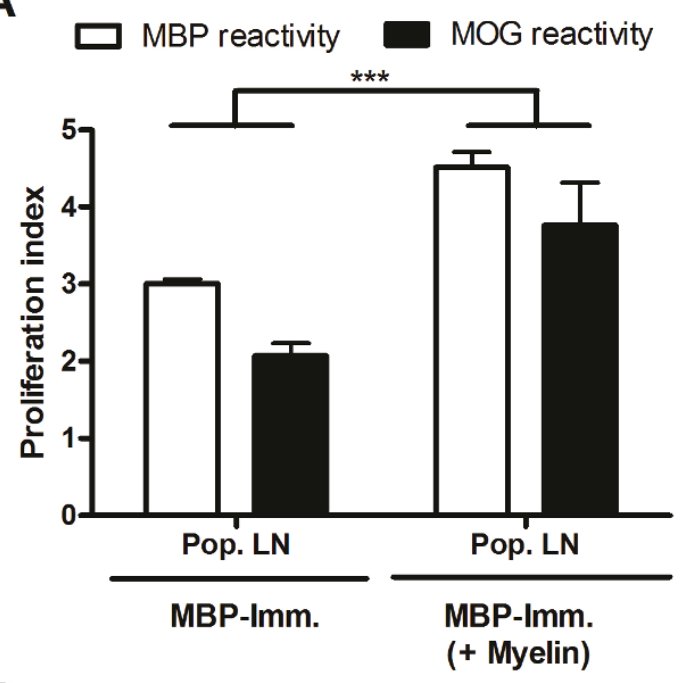

C
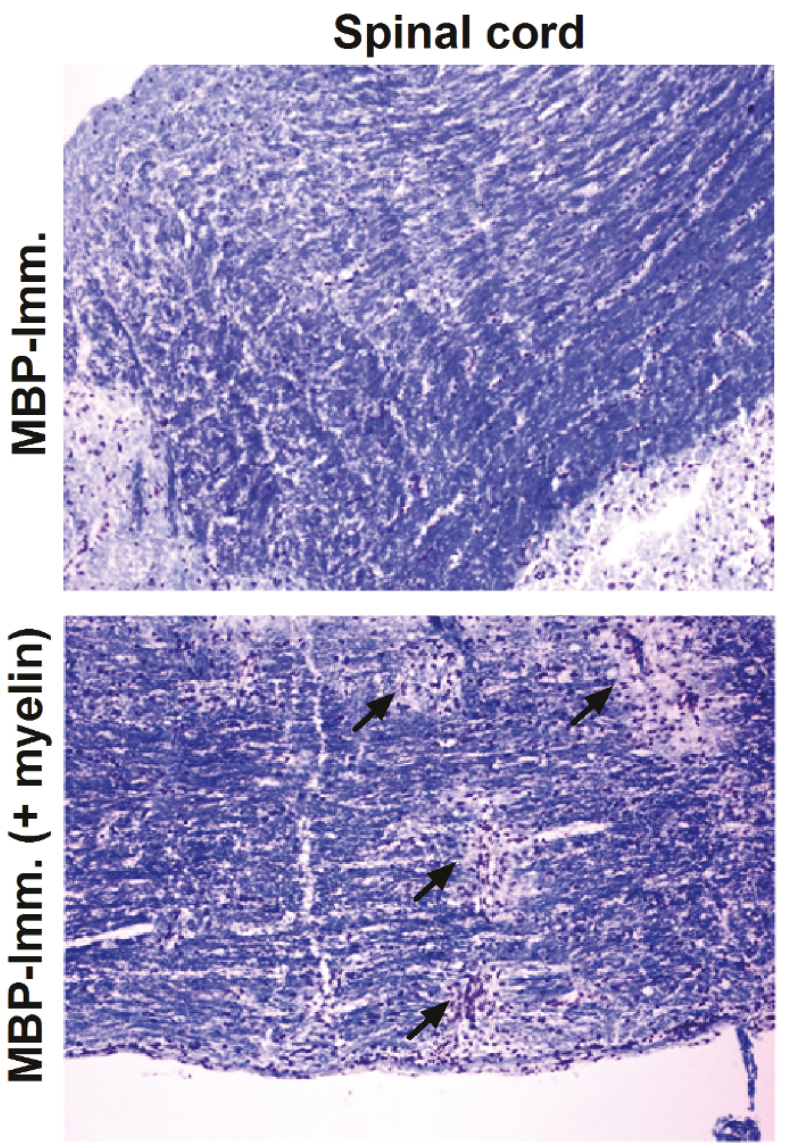

B

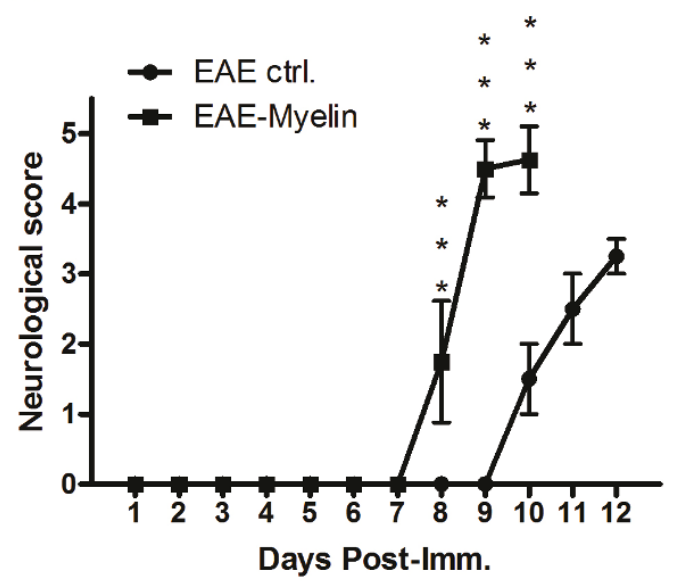

Brain
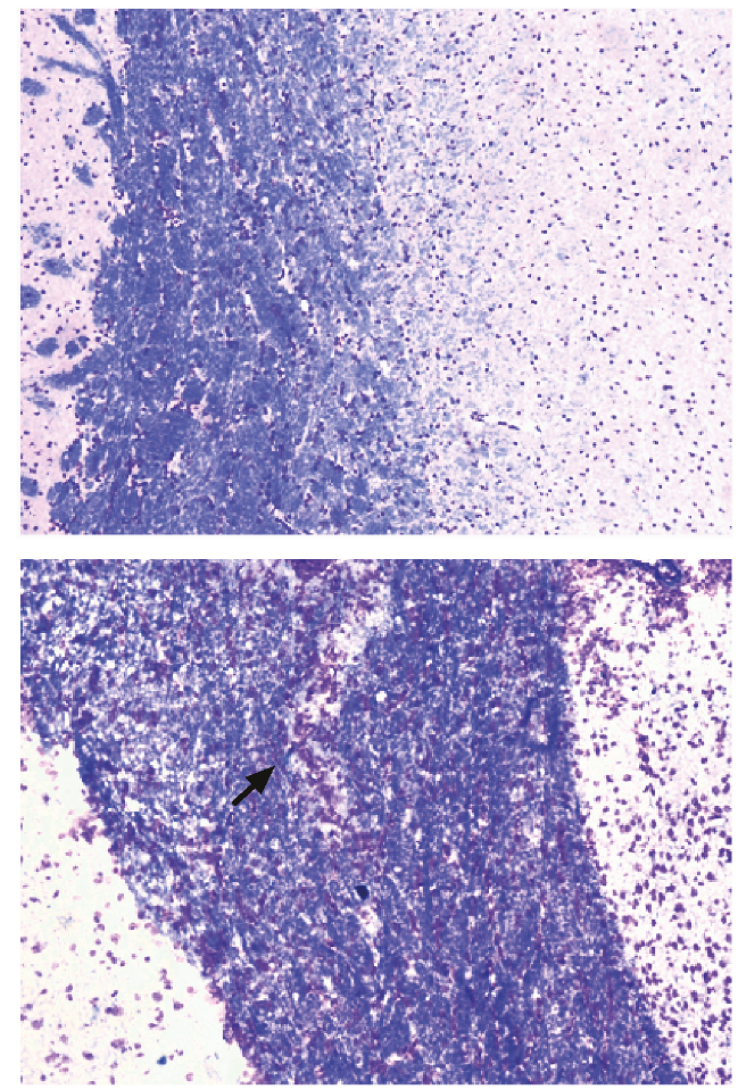

Figure 5 MBP-immunized animals demonstrate an aggravated disease course following myelin treatment. (a) MBP-immunized animals were treated with isolated myelin at day $-4,0,4$ and 8 (or left untreated). Ten days post-immunization popliteal lymph node cultures were isolated and MBP (white bars) and MOG (black bars) reactivity was assessed. Non-stimulated cultures were used as control. Data represent the mean of two independent experiments. (b) Neurological score of control $(N=5)$ and myelin-treated $(N=5)$, immunized animals was assessed daily. Due to severity of paralysis, myelin-treated animals were sacrificed at d10. (c) LFB/cresyl violet staining of spinal cord (left) and brain tissue (right) of myelin-treated animals at day 10. Arrows depict demyelinated regions. One experiment is shown. Pop. LN: Popliteal lymph node. 

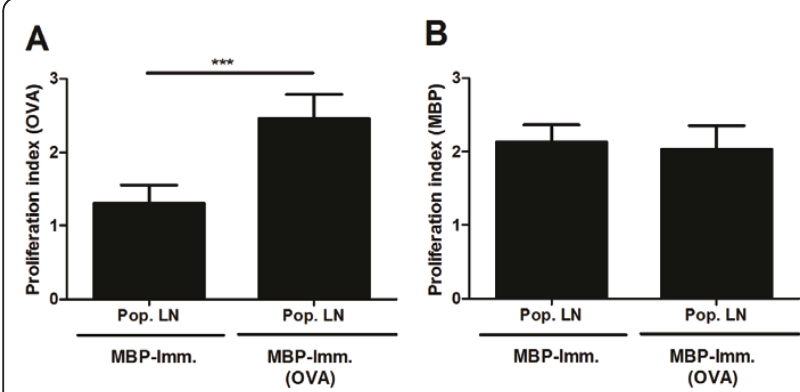

Figure 6 OVA does not influence lymphocyte reactivity to MBP in MBP-immunized animals. (a, b) MBP-immunized animals ( $N=$ 4) were treated with OVA at day $-4,0,4$ and 8 (or left untreated). Nine days post-immunization, OVA- (a) and MBP (b) reactivity of isolated lymph node cultures was assessed. Non-stimulated cultures were used as control. Data represent the mean of four independent experiments. Pop. LN: Popliteal lymph node.

particles and immune complexes, and activation of follicular B lymphocytes [57-60]. We demonstrate that a subcutaneous injection of myelin in the footpad results in a notable migration of myelin towards CD169 ${ }^{+}$ medullary and subcapsular sinus (SCS) macrophages in popliteal lymph nodes. Given the abundance of lipids in myelin, these results are in line with a recent report showing active phagocytosis of lipid-coated silica particles by SCS macrophages [61].

To explore the possible immune suppressive properties of mye-macrophages in vivo, OVA-immunized animals were treated subcutaneously in the footpad with myelin. Restimulated popliteal lymph nodes of myelintreated animals display reduced OVA-induced proliferation compared to lymphocytes derived from untreated OVA-immunized animals. This effect is independent of interference of myelin proteins on OVA antigen presentation, as lymph node cultures of MBP-immunized animals treated subcutaneously with OVA did not reduce MBP reactivity. These results demonstrate that myemacrophages suppress lymphocyte proliferation in vivo. In contrast, lymph node cultures derived from MBPimmunized animals that were treated with myelin showed an enhanced proliferative capacity. Although we demonstrated that mye-macrophages are unable to increase proliferation of MBP-reactive lymphocytes in vitro, the presence of other myelin-rich antigen-presenting cells, like migrated langerhans cells and local lymph node DCs, might explain the increased reactivity against MBP and MOG. Furthermore, B cells have been described to capture antigen-containing immune complexes from SCS macrophages processes and migrate to the $\mathrm{T}$ cell zone to influence antigen presentation $[57,60]$. Finally, the discrepancy in literature regarding the skewing of macrophages following myelin internalization suggests that myelin can have divergent effects on macrophage polarization and its APC-like or immune suppressive properties, which may depend on the macrophage origin and local environmental stimuli. Likewise, the nature of surrounding lymphocytes, for example being myelin-protein, non-myelin or myelinlipid specific, might determine whether the presence of mye-macrophages results in stimulation or suppression of lymphocyte activity. Future studies should therefore determine whether lymphocytes surrounding myemacrophages in CNS draining lymph nodes recognize antigen presented by these cells and are hereby activated.

Interestingly, we demonstrated an increased capacity of lymph nodes cells from myelin treated, OVA-immunized animals to produce NO following LPS stimulation. These results indicate a direct role of macrophage-produced $\mathrm{NO}$ in the observed decrease in OVA reactivity in myelin-treated animals, as observed in vitro. The importance of NO in the control of inflammation in EAE is supported by studies showing an aggravation or inability to recover following treatment with an iNOS inhibitor in respectively the induction or the remission phase of EAE $[62,63]$. Likewise, treatment with the NO-donor SIN-1 during the induction phase of EAE ameliorated EAE, which was correlated with a reduced immune cell infiltration and antigen-induced proliferation [64]. Finally, EAE insusceptibility in rat strains like the Piebald Virol Glaxo and the Brown Norway strain was correlated with an increased production of immune suppressive NO following immunization $[65,66]$. These results demonstrate that NO displays a disease-mitigating role in EAE by inhibiting lymphocyte proliferation. Based on these and our findings, we suggest that mye-macrophages in the perivascular space and CNS-draining lymph nodes can fulfill a suppressive role in MS by producing NO, hereby silencing autoreactive lymphocytes.

It is unclear which myelin components are responsible for the observed immune suppressive effects. To date, despite the abundance of lipids in myelin, most studies have mainly focused on the role of myelin proteins in neurodegenerative diseases. Interestingly, several lipids present in myelin have been reported to alter macrophage signaling and transcription. Intracellular, lipid sensors like LXR and PPAR, which are respectively activated by cholesterol derivates and non-esterified fatty acids, have recently been described as key regulators of lipid metabolism and inflammation, and may be activated following myelin internalization [67-69]. Similarly, individual lipids present in myelin can alter the macrophage or microglial response by binding to specific receptors and activating or blocking signalling cascades pivotal in inflammation [70-73].

Macrophages can adopt divergent phenotypes based on specific stimuli in their microenvironment [74-77]. 
Moreover, mye-macrophages have been described to display divergent phenotypes depending on the location in the lesion, suggesting that they are likely to exert diverse functions depending on their micro-location [12]. By using thioglycolate-elicited PECs, as a representative model for infiltrating monocytes in EAE and MS, we established that myelin internalization results in an altered macrophage function, characterized by an increased production of NO [78]. These mye-macrophages may have dual effects during MS pathogenesis. Whereas NO production by mye-macrophages can negatively influence neuronal integrity and block axonal conduction locally in the brain parenchyma, we show that NO also suppresses lymphocyte proliferation. Thus, depending on the surrounding cells, mye-macrophages can be involved in either limiting or promoting autoimmune-mediated demyelination.

\section{Conclusion}

We demonstrate that myelin phagocytosis leads to an altered macrophage function that inhibits lymphocyte proliferation. The observed immune suppression was mediated by an increased production of NO by myemacrophages. Additionally, we establish that myemacrophages fulfill an ambiguous role in vivo. On one hand mye-macrophages aggravate autoimmunity by activating myelin-reactive lymphocytes in secondary lymph nodes and on the other hand they suppress lymphocyte reactivity by producing NO.

\footnotetext{
Acknowledgements

We thank W. Leyssens for technical assistance. This work was supported by IWT Vlaanderen, FWO Vlaanderen, transnational University Limburg, Limburg
} Sterk Merk and Alma-in-Silico (EMR INT4.-1.3.-2008-03/003).

\section{Authors' contributions}

BJ performed the experiments, analyzed the data and wrote the manuscript. $\mathrm{HJ}, \mathrm{HN}$ and SP participated in its design and coordination, and have been involved in revising the manuscript. All authors have read and approved the final version of this manuscript

\section{Competing interests}

The authors declare that they have no competing interests.

Received: 15 March 2011 Accepted: 25 July 2011

Published: 25 July 2011

\section{References}

1. Hellings N, Raus J, Stinissen P: Insights into the immunopathogenesis of multiple sclerosis. Immunol Res 2002, 25:27-51.

2. Vanderlocht J, Hellings $\mathrm{N}$, Hendriks JJ, Stinissen P: The ambivalent nature of T-cell infiltration in the central nervous system of patients with multiple sclerosis. Crit Rev Immunol 2007, 27:1-13.

3. McFarland HF, Martin R: Multiple sclerosis: a complicated picture of autoimmunity. Nat Immunol 2007, 8:913-919.

4. Goverman J: Autoimmune T cell responses in the central nervous system. Nat Rev Immunol 2009, 9:393-407.

5. Trapp BD, Peterson J, Ransohoff RM, Rudick R, Mork S, Bo L: Axonal transection in the lesions of multiple sclerosis. N Engl J Med 1998, 338:278-285.
6. Huitinga I, van Rooijen N, de Groot CJ, Uitdehaag BM, Dijkstra CD: Suppression of experimental allergic encephalomyelitis in Lewis rats after elimination of macrophages. J Exp Med 1990, 172:1025-1033.

7. Huitinga I, Ruuls SR, Jung S, van Rooijen N, Hartung HP, Dijkstra CD: Macrophages in T cell line-mediated, demyelinating, and chronic relapsing experimental autoimmune encephalomyelitis in Lewis rats. Clin Exp Immunol 1995, 100:344-351.

8. Tran EH, Hoekstra K, van RN, Dijkstra CD, Owens T: Immune invasion of the central nervous system parenchyma and experimental allergic encephalomyelitis, but not leukocyte extravasation from blood, are prevented in macrophage-depleted mice. J Immunol 1998, 161:3767-3775.

9. Williams K, Ulvestad E, Waage A, Antel JP, McLaurin J: Activation of adult human derived microglia by myelin phagocytosis in vitro. J Neurosci Res 1994, 38:433-443.

10. Mosley K, Cuzner ML: Receptor-mediated phagocytosis of myelin by macrophages and microglia: effect of opsonization and receptor blocking agents. Neurochem Res 1996, 21:481-487.

11. van der Laan LJ, Ruuls SR, Weber KS, Lodder IJ, Dopp EA, Dijkstra CD: Macrophage phagocytosis of myelin in vitro determined by flow cytometry: phagocytosis is mediated by CR3 and induces production of tumor necrosis factor-alpha and nitric oxide. J Neuroimmunol 1996, 70:145-152.

12. Boven $L A$, van Meurs M, van Zwam M, Wierenga-Wolf A, Hintzen RQ, Boot RG, et al: Myelin-laden macrophages are anti-inflammatory, consistent with foam cells in multiple sclerosis. Brain 2006, 129:517-526.

13. Liu $Y$, Hao W, Letiembre $M$, Walter $S$, Kulanga $M$, Neumann $H$, et al: Suppression of microglial inflammatory activity by myelin phagocytosis: role of p47-PHOX-mediated generation of reactive oxygen species. $J$ Neurosci 2006, 26:12904-12913.

14. van Rossum D, Hilbert S, Strassenburg S, Hanisch UK, Bruck W: Myelinphagocytosing macrophages in isolated sciatic and optic nerves reveal a unique reactive phenotype. Glia 2008, 56:271-283.

15. Sun X, Wang X, Chen T, Li T, Cao K, Lu A, et al: Myelin activates FAK/Akt/ NF-kappaB pathways and provokes CR3-dependent inflammatory response in murine system. PLoS One 2010, 5:e9380.

16. Glim JE, Vereyken EJ, Heijnen DA, Vallejo JJ, Dijkstra CD: The release of cytokines by macrophages is not affected by myelin ingestion. Glia 2010, 58:1928-1936.

17. Piani D, Frei K, Do KQ, Cuenod M, Fontana A: Murine brain macrophages induced NMDA receptor mediated neurotoxicity in vitro by secreting glutamate. Neurosci Lett 1991, 133:159-162.

18. Flavin MP, Coughlin K, Ho LT: Soluble macrophage factors trigger apoptosis in cultured hippocampal neurons. Neuroscience 1997, 80:437-448.

19. Hendriks JJ, Teunissen CE, de Vries HE, Dijkstra CD: Macrophages and neurodegeneration. Brain Res Brain Res Rev 2005, 48:185-195.

20. Barnett MH, Henderson AP, Prineas JW: The macrophage in MS: just a scavenger after all? Pathology and pathogenesis of the acute MS lesion. Mult Scler 2006, 12:121-132.

21. Pineau I, Lacroix S: Proinflammatory cytokine synthesis in the injured mouse spinal cord: multiphasic expression pattern and identification of the cell types involved. J Comp Neurol 2007, 500:267-285.

22. Miller $\mathrm{E}$, Mrowicka M, Zolynski K, Kedziora J: [Oxidative stress in multiple sclerosis]. Pol Merkur Lekarski 2009, 27:499-502.

23. Nahrendorf M, Swirski FK, Aikawa E, Stangenberg L, Wurdinger T, Figueiredo $J$, et al: The healing myocardium sequentially mobilizes two monocyte subsets with divergent and complementary functions. J Exp Med 2007, 204:3037-3047.

24. Umemura N, Saio M, Suwa T, Kitoh Y, Bai J, Nonaka K, et al: Tumorinfiltrating myeloid-derived suppressor cells are pleiotropic-inflamed monocytes/macrophages that bear M1- and M2-type characteristics. J Leukoc Biol 2008, 83:1136-1144.

25. Morell P, Barrett CV, Mason JL, Toews AD, Hostettler JD, Knapp GW, et al: Gene expression in brain during cuprizone-induced demyelination and remyelination. Mol Cell Neurosci 1998, 12:220-227.

26. Arnett HA, Wang Y, Matsushima GK, Suzuki K, Ting JP: Functional genomic analysis of remyelination reveals importance of inflammation in oligodendrocyte regeneration. J Neurosci 2003, 23:9824-9832.

27. Li WW, Setzu A, Zhao C, Franklin RJ: Minocycline-mediated inhibition of microglia activation impairs oligodendrocyte progenitor cell responses 
and remyelination in a non-immune model of demyelination. $J$ Neuroimmunol 2005, 158:58-66.

28. Miller RH: Contact with central nervous system myelin inhibits oligodendrocyte progenitor maturation. Dev Biol 1999, 216:359-368.

29. Kotter MR, Zhao C, van Rooijen N, Franklin RJ: Macrophage-depletion induced impairment of experimental CNS remyelination is associated with a reduced oligodendrocyte progenitor cell response and altered growth factor expression. Neurobiol Dis 2005, 18:166-175.

30. Kotter MR, Li WW, Zhao C, Franklin RJ: Myelin impairs CNS remyelination by inhibiting oligodendrocyte precursor cell differentiation. J Neurosci 2006, 26:328-332.

31. Gredler V, Ebner S, Schanda K, Forstner M, Berger T, Romani N, et al: Impact of human myelin on the maturation and function of human monocytederived dendritic cells. Clin Immunol 2010, 134:296-304.

32. Sospedra M, Martin R: Immunology of multiple sclerosis. Annu Rev Immunol 2005, 23:683-747.

33. de Vos AF, van Meurs M, Brok HP, Boven LA, Hintzen RQ, van d V, et al: Transfer of central nervous system autoantigens and presentation in secondary lymphoid organs. J Immunol 2002, 169:5415-5423.

34. Fabriek BO, Zwemmer JN, Teunissen CE, Dijkstra CD, Polman CH, Laman JD, et al: In vivo detection of myelin proteins in cervical lymph nodes of MS patients using ultrasound-guided fine-needle aspiration cytology. J Neuroimmunol 2005, 161:190-194.

35. van Zwam M, Huizinga R, Melief MJ, Wierenga-Wolf AF, van Meurs M, Voerman JS, et al: Brain antigens in functionally distinct antigenpresenting cell populations in cervical lymph nodes in MS and EAE. J Mol Med 2009, 87:273-286.

36. Karman J, Ling C, Sandor M, Fabry Z: Initiation of immune responses in brain is promoted by local dendritic cells. J Immunol 2004, 173:2353-2361.

37. van Zwam M, Wierenga-Wolf AF, Melief MJ, Schrijver B, Laman JD, Boven LA: Myelin ingestion by macrophages promotes their motility and capacity to recruit myeloid cells. J Neuroimmunol 2010, 225:112-117.

38. Hendriks JJ, Slaets H, Carmans S, de Vries HE, Dijkstra CD, Stinissen P, et al: Leukemia inhibitory factor modulates production of inflammatory mediators and myelin phagocytosis by macrophages. J Neuroimmunol 2008, 204:52-57.

39. Norton WT, Poduslo SE: Myelination in rat brain: changes in myelin composition during brain maturation. J Neurochem 1973, 21:759-773.

40. Vandenbark AA, Gill T, Offner H: A myelin basic protein-specific T lymphocyte line that mediates experimental autoimmune encephalomyelitis. J Immunol 1985, 135:223-228.

41. Lescano-De-Souza A jr, Curi R: Cholesterol inhibits glutamine metabolism in LLC WRC256 tumour cells but does not affect it in lymphocytes: possible implications for tumour cell proliferation. Cell Biochem Funct 1999, 17:223-228.

42. Nishiyama A, Cavaglieri CR, Curi R, Calder PC: Arachidonic acid-containing phosphatidylcholine inhibits lymphocyte proliferation and decreases interleukin-2 and interferon-gamma production from concanavalin Astimulated rat lymphocytes. Biochim Biophys Acta 2000, 1487:50-60.

43. van der Veen RC, Dietlin TA, Dixon GJ, Gilmore W: Macrophage-derived nitric oxide inhibits the proliferation of activated Thelper cells and is induced during antigenic stimulation of resting $\mathrm{T}$ cells. Cell Immunol 2000, 199:43-49.

44. Peres CM, Otton R, Curi R: Modulation of lymphocyte proliferation by macrophages and macrophages loaded with arachidonic acid. Cell Biochem Funct 2005, 23:373-381.

45. Denham S, Rowland IJ: Inhibition of the reactive proliferation of lymphocytes by activated macrophages: the role of nitric oxide. Clin Exp Immunol 1992, 87:157-162

46. Bingisser RM, Tilbrook PA, Holt PG, Kees UR: Macrophage-derived nitric oxide regulates $\mathrm{T}$ cell activation via reversible disruption of the Jak3/ STAT5 signaling pathway. J Immunol 1998, 160:5729-5734.

47. Munn DH, Shafizadeh E, Attwood JT, Bondarev I, Pashine A, Mellor AL: Inhibition of T cell proliferation by macrophage tryptophan catabolism. $J$ Exp Med 1999, 189:1363-1372.

48. Rodriguez PC, Zea AH, DeSalvo J, Culotta KS, Zabaleta J, Quiceno DG, et al: L-arginine consumption by macrophages modulates the expression of CD3 zeta chain in T lymphocytes. J Immunol 2003, 171:1232-1239.

49. Rodriguez PC, Quiceno DG, Zabaleta J, Ortiz B, Zea AH, Piazuelo MB, et al: Arginase I production in the tumor microenvironment by mature myeloid cells inhibits T-cell receptor expression and antigen-specific Tcell responses. Cancer Res 2004, 64:5839-5849.

50. Zea AH, Rodriguez PC, Atkins MB, Hernandez C, Signoretti S, Zabaleta J, et al: Arginase-producing myeloid suppressor cells in renal cell carcinoma patients: a mechanism of tumor evasion. Cancer Res 2005, 65:3044-3048.

51. Mahidhara RS, Hoffman RA, Huang S, Wolf-Johnston A, Vodovotz Y, Simmons RL, et al: Nitric oxide-mediated inhibition of caspase-dependent T lymphocyte proliferation. J Leukoc Biol 2003, 74:403-411.

52. Ahvazi BC, Jacobs P, Stevenson MM: Role of macrophage-derived nitric oxide in suppression of lymphocyte proliferation during blood-stage malaria. J Leukoc Biol 1995, 58:23-31.

53. Albina JE, Abate JA, Henry WL Jr: Nitric oxide production is required for murine resident peritoneal macrophages to suppress mitogenstimulated T cell proliferation. Role of IFN-gamma in the induction of the nitric oxide-synthesizing pathway. J Immunol 1991, 147:144-148.

54. Isobe K, Nakashima I: Feedback suppression of staphylococcal enterotoxin-stimulated T-lymphocyte proliferation by macrophages through inductive nitric oxide synthesis. Infect Immun 1992, 60:4832-4837.

55. Shimizu T, Cai S, Tomioka H: Roles of reactive nitrogen intermediates and transforming growth factor-beta produced by immunosuppressive macrophages in the expression of suppressor activity against T cell proliferation induced by TCR stimulation. Cytokine 2005, 30:7-13.

56. Hamilton MJ, Antignano F, von RA, Boucher JL, Bennewith KL, Krystal G: TLR agonists that induce IFN-beta abrogate resident macrophage suppression of T cells. J Immunol 2010, 185:4545-4553.

57. Carrasco YR, Batista FD: B cells acquire particulate antigen in a macrophage-rich area at the boundary between the follicle and the subcapsular sinus of the lymph node. Immunity 2007, 27:160-171.

58. Junt T, Moseman EA, lannacone M, Massberg S, Lang PA, Boes M, et al: Subcapsular sinus macrophages in lymph nodes clear lymph-borne viruses and present them to antiviral B cells. Nature 2007, 450:110-114.

59. Phan TG, Grigorova I, Okada T, Cyster JG: Subcapsular encounter and complement-dependent transport of immune complexes by lymph node B cells. Nat Immunol 2007, 8:992-1000.

60. Phan TG, Green JA, Gray EE, Xu Y, Cyster JG: Immune complex relay by subcapsular sinus macrophages and noncognate $B$ cells drives antibody affinity maturation. Nat Immunol 2009, 10:786-793.

61. Barral P, Polzella P, Bruckbauer A, van Rooijen N, Besra GS, Cerundolo V, et al: CD169(+) macrophages present lipid antigens to mediate early activation of iNKT cells in lymph nodes. Nat Immunol 2010, 11:303-312.

62. O'Brien NC, Charlton B, Cowden WB, Willenborg DO: Nitric oxide plays a critical role in the recovery of Lewis rats from experimental autoimmune encephalomyelitis and the maintenance of resistance to reinduction. $J$ Immunol 1999, 163:6841-6847.

63. Kahl KG, Zielasek J, Uttenthal LO, Rodrigo J, Toyka KV, Schmidt HH: Protective role of the cytokine-inducible isoform of nitric oxide synthase induction and nitrosative stress in experimental autoimmune encephalomyelitis of the DA rat. J Neurosci Res 2003, 73:198-205.

64. Xu LY, Yang JS, Link H, Xiao BG: SIN-1, a nitric oxide donor, ameliorates experimental allergic encephalomyelitis in Lewis rats in the incipient phase: the importance of the time window. J Immunol 2001, 166:5810-5816

65. Cowden WB, Cullen FA, Staykova MA, Willenborg DO: Nitric oxide is a potential down-regulating molecule in autoimmune disease: inhibition of nitric oxide production renders PVG rats highly susceptible to EAE. $J$ Neuroimmunol 1998, 88:1-8.

66. Staykova MA, Paridaen JT, Cowden WB, Willenborg DO: Nitric oxide contributes to resistance of the Brown Norway rat to experimental autoimmune encephalomyelitis. Am J Pathol 2005, 166:147-157.

67. Valledor AF, Ricote M: Nuclear receptor signaling in macrophages. Biochem Pharmacol 2004, 67:201-212.

68. Hong C, Tontonoz P: Coordination of inflammation and metabolism by PPAR and LXR nuclear receptors. Curr Opin Genet Dev 2008, 18:461-467.

69. Bensinger SJ, Tontonoz P: Integration of metabolism and inflammation by lipid-activated nuclear receptors. Nature 2008, 454:470-477.

70. Wang PY, Kitchens RL, Munford RS: Phosphatidylinositides bind to plasma membrane CD14 and can prevent monocyte activation by bacterial lipopolysaccharide. J Biol Chem 1998, 273:24309-24313. 
71. Jou I, Lee JH, Park SY, Yoon HJ, Joe EH, Park EJ: Gangliosides trigger inflammatory responses via TLR4 in brain glia. Am J Pathol 2006, 168:1619-1630.

72. Jeon SB, Yoon HJ, Park SH, Kim IH, Park EJ: Sulfatide, a major lipid component of myelin sheath, activates inflammatory responses as an endogenous stimulator in brain-resident immune cells. J Immunol 2008 181:8077-8087.

73. Lee C, An HJ, Kim JL, Lee H, Paik SG: Inhibitory effect of a phosphatidyl ethanolamine derivative on LPS-induced sepsis. Mol Cells 2009, 27:251-255.

74. Mantovani A, Sica A, Locati M: Macrophage polarization comes of age. Immunity 2005, 23:344-346.

75. Gordon S, Taylor PR: Monocyte and macrophage heterogeneity. Nat Rev Immunol 2005, 5:953-964.

76. Mosser DM, Edwards JP: Exploring the full spectrum of macrophage activation. Nat Rev Immunol 2008, 8:958-969.

77. Martinez FO, Helming L, Gordon S: Alternative activation of macrophages: an immunologic functional perspective. Annu Rev Immunol 2009, 27:451-483.

78. Ghosn EE, Cassado AA, Govoni GR, Fukuhara T, Yang Y, Monack DM, et al: Two physically, functionally, and developmentally distinct peritoneal macrophage subsets. Proc Natl Acad Sci USA 2010, 107:2568-2573.

doi:10.1186/1742-2094-8-85

Cite this article as: Bogie et al: Myelin-phagocytosing macrophages modulate autoreactive T cell proliferation. Journal of Neuroinflammation $20118: 85$

\section{Submit your next manuscript to BioMed Central and take full advantage of:}

- Convenient online submission

- Thorough peer review

- No space constraints or color figure charges

- Immediate publication on acceptance

- Inclusion in PubMed, CAS, Scopus and Google Scholar

- Research which is freely available for redistribution

Submit your manuscript at www.biomedcentral.com/submit 